\title{
Is the Writing on the Wall for Tabletops?
}

\author{
Nadia Pantidi, Yvonne Rogers, and Hugh Robinson \\ Computing Department \\ Open University \\ Milton Keynes \\ MK7 6AA, UK
}

\begin{abstract}
We describe an ethnographic study that explores how low tech and new tech surfaces support participation and collaboration during a workshop breakout session. The low tech surfaces were post-it notes and large sheets of paper. The new tech surfaces were writeable walls and a multi-touch tabletop. Four groups used the different surfaces during three phases: i) brief presentation of position papers and discussion of themes, ii) the creation of a group presentation and iii) a report back session. Participation and collaboration varied depending on the physical, technological and social factors at play when using the different surfaces. We discuss why this is the case, noting how new shareable surfaces may need to be constrained to invite participation in ways that are simply taken for granted because of their familiarity when using low tech materials.
\end{abstract}

Keywords: Collaboration, equitable participation, interactive tabletops, workshops.

\section{Introduction}

Workshops are commonplace in education and business, providing an occasional forum for groups of people to discuss and share ideas. Typical goals of a workshop include establishing a new area, identifying key themes, and addressing new concerns. The extent to which a workshop is successful in achieving its aims, however, depends on a number of factors. These include ensuring everyone has an opportunity to participate and express their views [21]. One method that is commonly employed is to break out into smaller groups for certain sessions of the workshop, with the specific intention of providing more opportunities for participants to contribute in a smaller setting. The sub-groups typically work on a specific task, such as identifying a set of requirements for a new research area and then reporting back to the larger group to share their ideas.

A common practice used to facilitate participation within a breakout group is to provide a variety of low tech materials that group members can externalize and share their ideas on (e.g., post-it notes, sheets of papers and flipcharts). Group members can write their own ideas, opinions or questions down first on individual post-its and then compile them into a meaningful group structure using a shared flipchart. Recently, 'new tech' surfaces have been experimented with, such as interactive tabletops and special writeable walls. An assumption is that because of their shareable properties (e.g., they can be interacted with simultaneously by those standing or sitting around 
them) they will promote more equitable participation and greater awareness of what others are saying and doing. However, it is unclear if this is the case for meetings, where the goal is for groups of individuals to collectively generate and explore ideas together, assemble and agree on them and subsequently present the outcomes to a wider group.

Our research is concerned with how different kinds of surfaces support the creation and sharing of ideas in breakout groups, where equitable participation is a desirable outcome. Specifically, we describe an ethnographic study that explored how different low and new tech surfaces were used by breakout groups to work together, initially to discuss ideas, then generate themes and finally to present these back to the wider meeting. Our study revealed marked differences between the groups in terms of the participants' contribution and the way they progressed with the task. We discuss these findings in terms of how the different technologies mediate collaboration, in terms of how participants approach and appropriate the different surfaces, the extent of participation, the nature of the turn-taking that took place and how the technologies can be exploited by gatekeepers in a group.

\section{Background}

Low tech materials, such as paper and post-it notes, are frequently used to support collaborative activities, especially early design phases [2]. They are also used for collaborative planning in medical settings [e.g., 10, 13], the military [19] and control centres [16]. Their advantages are well known and have been written about extensively; for example, paper is cheap, lightweight, intuitive to use, robust and can be easily manipulated and shared [3, 32]. Physical artifacts, such as annotated notes, are also highly tangible. They may be used as external thinking props to explain a principle, an idea or a plan to others in the group. The persistence and ability to manipulate physical artifacts may also result in a diversity of options being explored in a group setting.

Software tools have been developed for use in structured meetings to facilitate and record the discussions that occur such, as those that take place in the planning of NASA's mission control. They have been used to integrate notes, visualise various forms of pre-stored data and to annotate plans and ideas. Examples include Debategraph [7] and Compendium [33] which capture and represent information and argumentation. Interactive whiteboards have also been used to support focused meetings around a single issue [25]; ongoing, continuous work across a host of domains [22] and small group collaboration in informal meetings [30].

Several studies investigating how interactive displays are used by people in collaborative settings have examined the context in which they are initially approached. The presence of social and visual cues that invite people to use them has been found to be critical $[1,5,6,11]$. How comfortable people feel knowing that their actions and their effects on a shared display are highly visible to others in a group setting may also affect their willingness to participate. Such self-consciousness can deter people from taking part in a group activity [4]. For example, people needed constant encouragement and demonstration to interact with a shared whiteboard system called Plasma Poster [5]. User studies have also shown how actual use of interactive displays can be 
quite different from their intended use [e.g., 4, 15, 18, 24]. When the shared display is vertical, as is typically the case with whiteboards, people work around them in somewhat unnatural and uncomfortable ways [9]. This can make it difficult for participants to take over control or for them to hand it over to others. The effect can be suboptimal communication of ideas and activity progression. One strategy people use is to monitor the current speaker or user and explicitly orient themselves in order to find when it is possible to take the floor $[31,26]$. Similarly, the speaker or user can help by making his/her withdrawal visibly noticeable.

In contrast to vertical displays, horizontal tabletop displays have been designed so that more than one person can use them simultaneously [27]. Examples include Microsoft's Surface [20] and MERL's DiamondTouch [8]. An assumption is that they can support more flexible forms of collaboration because they provide more opportunities for interaction and the switching of control between group members. People seem more willing to interact with multi-touch tabletops in the presence of others despite their actions being highly visible and observable by others in the group [11]. This may be because these kinds of shared horizontal surfaces lure people to touch them without feeling intimidated or embarrassed by the consequences of their actions. The familiar and lightweight action of touching a surface may also make it easier for people to take part in a social/public setting. User studies have shown how groups of people, new to tabletops, find it easy and enjoyable when sharing and assembling of sets of digital images for a variety of collaborative tasks $[12,28]$. However, studies of whether this new form of shared interaction promotes more equitable participation and less awkward ways of taking control in small groups have been mixed. Marshall et al. [17] and Rogers et al. [28] have found that multi-touch tabletops supported more equitable participation in terms of group member's digital interactions but that their verbal contributions remained uneven. Dominant people continued to speak the most while shy and non-native speakers spoke the least.

Ideally, these new kinds of shareable technologies should be designed to allow groups comfortably and easily to access, create, interact with and move digital content in an equitable and free-flowing manner. However, the extent to which these goals can be met depends on a number of factors, including how obvious it is to the group members to know what to do at an interface and how to take turns to progress with a collaborative task. Our research is concerned with how different technologies (tabletop, wall, flipcharts, post-its) invite people to generate, add, manipulate or structure ideas from a shared display. In particular, we are interested in whether groups use these new tech surfaces in equitable and collaborative ways and how this compares with the appropriation of more familiar low tech materials.

\section{The Study}

To examine how low tech and new tech surfaces affect group participation, especially the creation, sharing and organization of ideas we conducted an ethnographic study of an academic two-day workshop, where the organizers had decided to provide four breakout groups with different kinds of technologies. The workshop took place in a technologically augmented space, Qspace, an adaptable space with an emphasis on creativity. To begin, we describe our methodological approach, the setting and the way the breakout session was setup in terms of the groups and the surfaces. 


\subsection{The Method}

The methodological approach employed in our study was ethnographic, involving observations of naturally occurring activities and semi-structured interviews. The workshop was planned and run by organizers who we were acquainted with but our involvement with the event was purely as participant observation. Hence, we had no influence over the design of the breakout groups or the materials used, but observed it through the eyes of those who inhabited it. Following the ethnographic approach, all activities observed were considered as 'strange'; no preliminary hypotheses were formed beforehand and no particular feature of use or interaction was given a priori significance. The collected data consisted of field notes, photos, documents and audio recordings from participant observation and semi-structured interviews. We provide incidents, activities and practices within their context, to emphasise that their meaning is properly comprehended within the appropriate social context.

\subsection{The Setting}

Qspace is a Centre of Excellence in Teaching and Learning (CETL) space that was created as part of Higher Education Funding Council for England (HEFCE) joint initiative between two universities. It was envisioned to be a technologically rich but not technology-driven learning space that was quite different from the constraints of a traditional lecture hall or seminar room. It provides both physical and technological resources that can be used in a variety of configurations with the purpose of supporting innovative creative processes. The space has been designed to create a relaxed atmosphere with all white moving walls, in which multi-coloured LEDs, curtains, bean bags, numerous projectors and PLASMA screens have been placed. Some of the walls have been customized as writeable surfaces. For the workshop, a DiamondTouch tabletop was placed into the space by the workshop organisers. Qspace, therefore, is a rather untypical space for an academic workshop, especially for paper presentations. On the other hand, the breakout sessions could be designed flexibly by using different configurations of technology and physical space.

\subsection{Groups and Materials}

During initial planning meetings between the managers/facilitators of QSpace and the workshop organisers an explicit suggestion was made to introduce a more 'creative' activity such as building conceptual representations with low tech materials (clay, polyethylene, cardboard), as this would match the QSpace 'owners' criteria of use ${ }^{1}$. It is also an activity that has been found to be successful in the past for that space. The workshop organisers incorporated this suggestion by providing groups with different surfaces to work on during a breakout session. From their perspective, they were

${ }^{1}$ Before the facilitators allow any activity to take place in the space, they were going through a list of criteria that the activity should match up to some degree. These criteria ensure that the activity is well suited for the space and complies with Qspace's evaluation pre-requirements in terms of its funding. 
Table 1. Types of surface used in each breakout group

\begin{tabular}{|l|l|l|l|l|}
\hline & Group A & Group B & Group C & Group D \\
\hline Surface & Post-its & $\begin{array}{l}\text { Large sheets } \\
\text { of paper }\end{array}$ & Writeable wall & Tabletop \\
\hline
\end{tabular}

interested in whether and how the different surfaces would affect collaboration within the groups.

For the breakout session that was observed during the study, four groups were created (A, B, C and D), each consisting of 9-10 participants. The organisers assigned one person in each group to initially present the group's position papers in a summarized version as a starting point for a discussion. The next phases involved them creating a presentation of the main discussion themes as a concept map that they would later present back to the other groups. Each group was given either low or new tech surfaces to create their presentation on (see Table 1). Group A was provided with different sized post-its and coloured markers; Group B was provide with large sheets of paper and coloured markers; Group $\mathrm{C}$ was given coloured markers for writing on the special wall surface and Group D was provided with the tabletop that displayed a simple concept map tool for typing in their ideas and arranging into a particular structure. In terms of our classification of technology, Groups A and B were considered as low tech and Groups C and D as new tech.

\section{Findings}

Observations and interviews with the participants revealed differences between the groups in terms of how they approached and appropriated the different surfaces and the turn-taking that took place. A main finding was that the extent and kind of collaboration and participation varied across the four groups. Roles, such as scribe, were adopted by or assigned to individuals and were determined to some extent by the kind of surface used. Below we describe the findings in terms of, firstly, how participants approached and appropriated the different surfaces; secondly, the turn-taking that took place; thirdly, the extent to which equitable participation took place and fourthly, whether gatekeeping emerged in a group and the role the technology played in enabling it to materialize.

\subsection{Approaching and Appropriating the Different Surfaces}

In the low tech groups, the post-it notes were handed out early on to the participants. As expected, Group A wrote their own ideas down on the big post-its. The group shared the big post-its and used them as if they were small ones (figure 1a) rather than using them as a poster surface and adding small post-its notes to it. One of the members (A1) commented: "From the [participant's] presentation [of the position papers] we recognized the general problem area that we were to address. We decided that we should choose some aspects to address more specifically and we did sort of an informal brainstorming (...) For that, each one of us wrote on a post-it [a big one] issue or idea about the area (...)" . 
In Group B, after only a few minutes of discussion, one of the participants (B1) suggested they start working on their presentation while still discussing the ideas. $\mathrm{He}$ also requested from the organizers that they be given post-its rather than begin writing their ideas on the large sheets of paper. This suggests that he wanted them to write their own ideas down first and then move them onto a shared display - rather than try to write them straight onto the paper. It also meant they could move the ideas around which would not be possible if written directly onto the paper. As the session progressed, B1 placed one of the large pieces of paper on the floor in the middle of the group and invited the others to start writing on the post-its important issues that arose from the discussion.

Both Groups A and B took a photo of their shared map of post-it ideas and presented it using one of the data projectors to the rest of the groups in the report back session. Hence, the second phase was integrated with the first, meaning that the groups were discussing and creating their ideas at the same time. Prior familiarity with the paper-based surfaces resulted in them having no problems writing their ideas on them and then assembling them into a shared structure. All of them readily understood that the post-its were intended for writing a comment, idea or question on and afterwards making it public by reading it or sticking it on the flip chart paper or the wall.

In Group $\mathrm{C}$ one participant began by projecting slides of the main points of the position papers on the wall. The group then discussed their ideas. Only towards the end of the session did one or two of them approach the writeable wall and write up their ideas. Hence, the assembling and explication of ideas was left to the end of the session rather than being a shared integrated activity throughout - as was the case in the low tech groups. One of the participants commented: "(...) it was as if we were mesmerized by the screen and the presentation and didn't want to move [on to the next stage]". The participants who wrote on the wall also wrote lists of points rather than create a concept map. One participant noted that their initial ideas were too small and illegible from far away and so she rewrote these to be neater and bigger on an adjacent wall so that a larger audience could read them. Thus the transition from the generation and sharing of ideas in the sub-group to the reporting back to the whole workshop was much more cumbersome than in the low tech groups. Part of the problem may have been because the group was provided with the same surface for the generation and the reporting back of ideas. It was only when they realized this that they wrote them up in a form that could be viewed from a wider audience.

For Group D, a simple interactive concept map was developed for use in conjunction with the tabletop surface. The participants were instructed on how use it by the person who had designed the concept map software (D1). He explained how to create nodes, type text and build up a concept map. It was observed that the group chose not to sit around the tabletop at the beginning (see figure 1b); instead, they approached the tabletop later on only after they were explicitly asked by D1 to do so. Hence, similar to Group C, and in contrast with the familiar post-its and paper sheets, the participants appeared to be more reluctant in the beginning to use the tabletop concept map. It required scaffolding and encouragement by the software developer. D1 then took a screen shot of the resultant concept map and projected it onto the wall during the subsequent feedback session. 


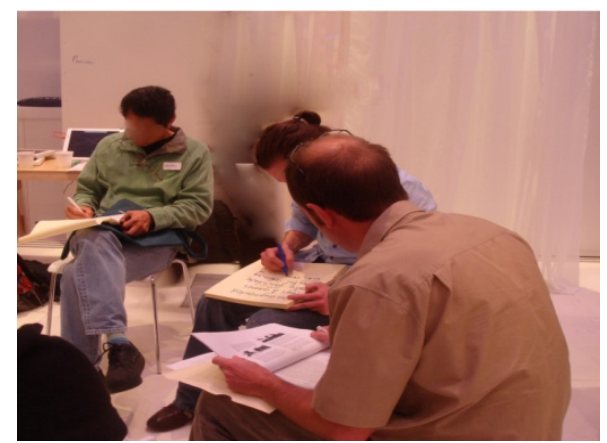

Fig. 1a. Writing on the big post-it notes

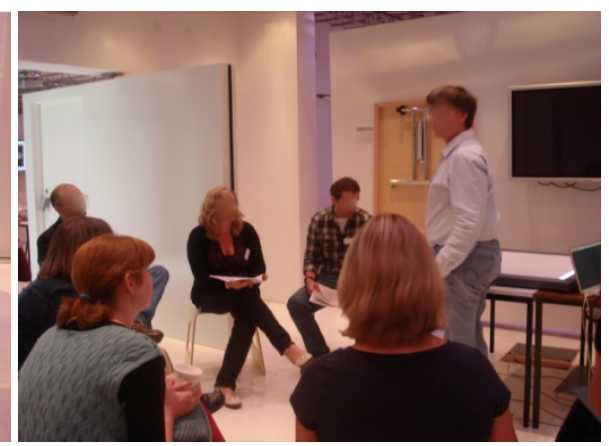

Fig. 1b. Group D sitting next to the tabletop

\subsection{Turn-Taking in Conversation and Writing Up of Ideas}

Turn-taking was evident in all groups during the discussion phase, although the extent of contribution differed between the groups. In each group, the occasional speaker paused after making his/her point, providing an opportunity for another participant to comment or make another point. Some breakdowns, such as interrupting the speaker and simultaneous speaking were observed but were not seen as out of the ordinary.

When it came to writing up the ideas generated in the discussion for the subsequent presentation to the whole workshop there was some evidence of turn-taking in Group A (post-its) but less so in the other groups. Members of Group A tried to place the post-its on the wall but they did not stick on the surface. They then resorted to writing their ideas up on the wall, where they all took turns to write up what was on the postits by using pen markers. In contrast, Group C split into three sub-groups that worked on separate themes and where only one person from each sub-group took charge of writing their notes on the wall (figure $2 b$ ). There was some negotiation between the members of each sub-group about the written content but the members did not take turns in the writing. A similar situation was observed in Group B where one person took charge of making a concept map during the whole session and where no turntaking occurred (figure 2a). A pattern of parallel work was also observed in Group D where four members worked largely by themselves to type in text and create nodes (figure 2c). Even though three of them stepped back to enable the other group members to have a go, only one out of the five remaining members took the opportunity to do so. It appears that in contrast to Group A, these three groups adopted a way of working that is more commonly observed in "whiteboard interaction scenarios"2.

\subsection{Extent of Contribution}

The extent each member contributed to the task varied across groups and phases of the task. In Group A, all members of the group contributed when discussing and

\footnotetext{
${ }^{2}$ By "whiteboard interaction scenario" is meant a situation where one person stands in front of the whiteboard and writes on it while the rest of the group makes only verbal contributions. In such cases, it is possible that some group members may not contribute at all.
} 


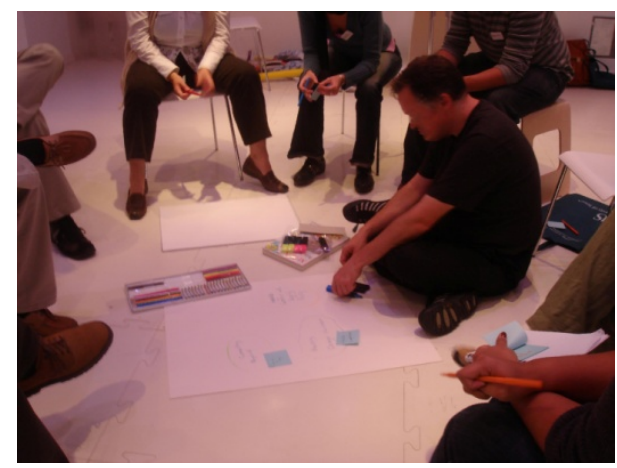

Fig. 2a. B1 in charge of creating the concept map

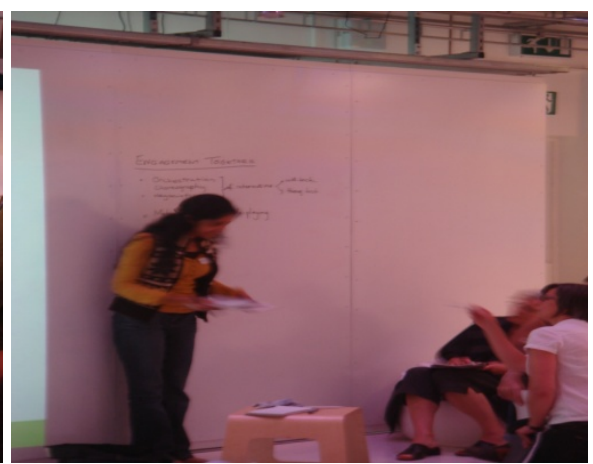

Fig. 2b. The wall used as a big whiteboard

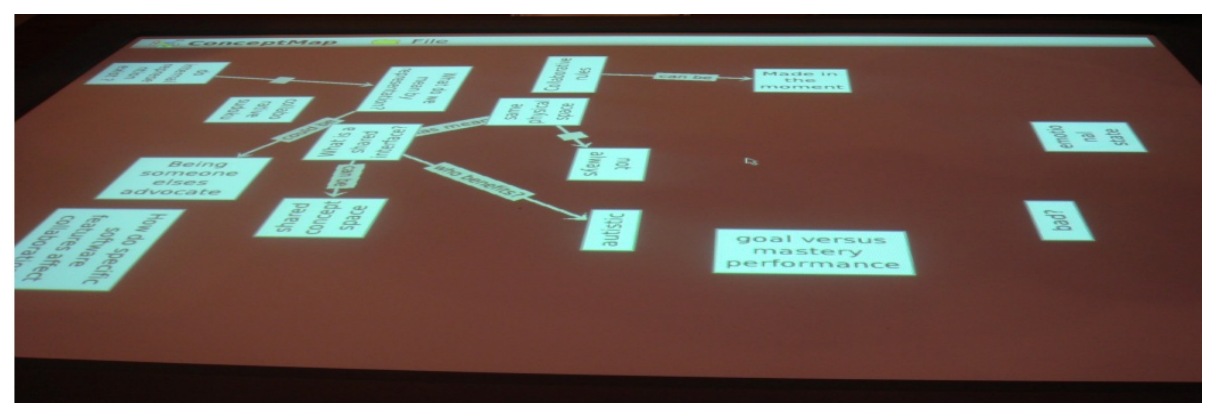

Fig. 2c. The tabletop concept map: the nodes that are in the centre are the ones created by D1 as starting point while the directionality of the nodes typed indicates parallel individual work

writing on the post-its and then when writing these up on the walls. In Group B each participant contributed to the task by writing at least one post-it but not all took part in placing the post-its and assembling the concept map. Moreover, it was observed that not all members participated equally in the discussion. This observation is also supported by a record of verbal contributions that one member of the group sketched during the session, where each participant was represented by a node and was checked every time he/she contributed to the discussion. The majority of the participants were checked once or twice and only two had multiple checks for verbal contributions. Only two participants were observed to take a leading role: B1 and B2. B1 can be described as the "doer" and B2 as the "talker" based on their contributions.

In Group C, although one participant was in charge of writing and no physical turntaking took place, our observations indicate that there was constant verbal negotiation between the members of the sub-groups about the content of the final result. The participant holding the marker neither wrote exclusively his/her ideas nor was a simple listener to the suggestions of others. In some instances the group was even negotiating about details such as the appropriate phrasing and not just the content of the text. In Group D, all members contributed during the discussion. When the move to the tabletop took place, however, only five out of the nine people followed; the other four 
continued with the discussion during the rest of the session. Of the 4 participants who moved to the tabletop, only two remained for any length (one being D1). One of them when asked later, why, provided a range of reasons: "I had to move from one area to another and we [meaning the four participants] were discussing... I don't even remember what we were discussing... what I remember is that I felt intimidated and that it [adding text to the surface] is more permanent than the discussion <pause $>$ I also felt more conscious that I was the most junior one". A possible factor that appears to have affected the extent to which members of this group made a contribution was their status in the group and how comfortable they felt about making public their ideas in front of others, who knew more. This did not appear to happen in the other groups presumably because they were less intimidating to use in the presence of others.

\subsection{Gatekeeping}

The inequality in participation across the groups can also be explained in part by gatekeeping, where "actors have control over key sources or avenues of opportunity. Gatekeepers exercise control at and during key phases” [12]. This was most evident in Groups B and D, but not in Groups A and C. B1 and D1 took control of the materials that restricted others from participating at various stages. B1 was always in charge of the post-its and the concept map creation (figure 2a). He took over the manipulation of these almost from the beginning of the session by handing out the postits, suggesting how to work, taking the poster paper and placing it on the floor and positioning himself next to it. His assertive behavior constrained the form of collaboration that could take place for the rest of the session. He implicitly assigned roles to the other participants that were limited to verbal and not physical contribution at least in terms of the concept map task.

D1 was in a different position than B1, as he had programmed the concept map software. From this point of view, it was clear that he possessed knowledge on the tool that no other participant had and in that sense he was an obvious gatekeeper. However, the rest of the group who were familiar with tabletops could have overcome this obstacle with some help from him. This did not happen and other more implicit signs contributed to his being established as a gatekeeper and thus inhibiting other participants from interacting with the tabletop. At some point during the discussion phase, D1 left the group circle and moved to the tabletop where he started typing text and creating nodes for the concept map (figure 3a). As D1 explained later in an interview he wanted to make some examples that would be of use when he explained to the group how to use the software. However, this action, viewed from the other participants, implied his right and ownership over the tabletop that they did not share. Similarly, during the concept map task, D1's presence and guidance was continuous even when he had supposedly stepped down; he was leaning towards the tabletop commenting, assisting and making sure everything was working properly (figure $3 \mathrm{~b}$ ). Moreover, when everyone from the group had finished working he added some final touches by re-organising parts of the concept map.

It was also noted how the gatekeepers in Groups B and D maintained that role in the report back session to the rest of the workshop. Hence, their role of gatekeeper was able to be preserved, transferred and extended beyond the physical or digital manipulation of the tools. 


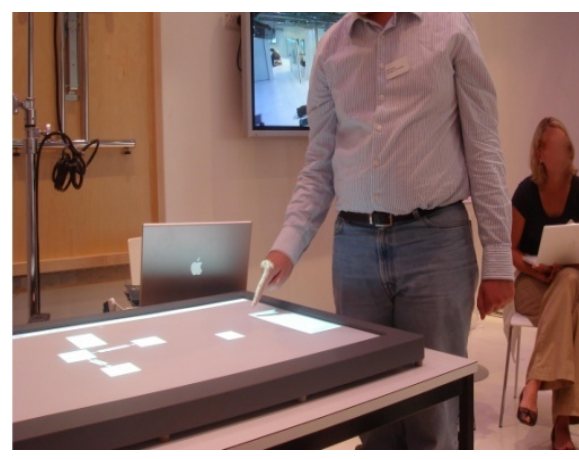

Fig. 3a. D1 typing on the tabletop while the rest of the group is discussing

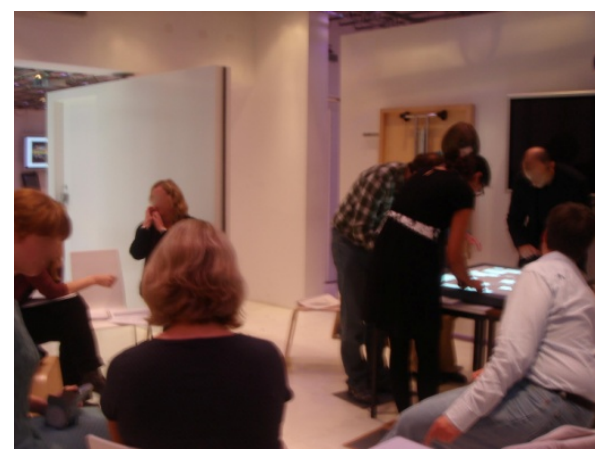

Fig. 3b. D1 leaning towards the tabletop

\section{Discussion}

The findings from our ethnographic study reveal how the type of surface that is provided to support the generation and sharing of ideas can affect the nature of the collaboration and interactions that take place during a breakout group. While all the groups were able to complete the task using a particular technology, it was the ubiquitous, low tech post-it note that turned out to be the most democratic medium; each member of the group was able to contribute their ideas through using it, which they then took turns to combine into a shared representation as written points on the wall. The two novel technologies - the writeable wall and the tabletop - were used in less equitable ways. In particular, they were not used until the end of the breakout session and then only by a few members of the group. One reason for this is that the actions of writing on a wall or entering text into existing software node structures are unfamiliar to the participants and which they feel awkward initially approaching.

Although writing is a very familiar activity, writing on a wall is not. It is also unnatural for a group of 8-10 people to stand around together at a wall and write together. Hence, it is not surprising that Group C broke up into smaller groups and where each allocated a scribe to write up their ideas on the wall. However, the result was that three streams of ideas generated by the sub-groups were not integrated in the way they were in the low tech groups. Similarly, although the participants in Group D were familiar with multi-touch surfaces, they had not created a concept map together by entering their ideas into a software tool. As it was not possible for all group members to take part at the same time at the tabletop (it was constrained for only 4 people to use) it was, likewise, not surprising that they broke up into two sub-groups. However, the sub-groups did not switch roles despite those at the tabletop inviting them to do so, mid-way through. This resistance to switching places when using such technologies is redolent of the fixing of roles that occur when groups work around whiteboards [24]. Typically, one person is in charge and the rest contribute verbally and not in equal terms, such as was found in Whittaker and Schwarz's [34] study; the advantage of using a physical wall compared to a PC-based software wall was attributed to it being collaboratively constructed whereas the data was entered into the software tool and thus 'owned' exclusively by the project manager. 
In these settings, certain members of a group can unwittingly or explicitly turn into gatekeepers. As found in Group D, one individual took control throughout in terms of the creation of the ideas, their organization and then the subsequent presentation of them to the rest of the workshop. However, the same also happened in one of the low tech groups, where B1 took control of the situation and effectively became a leader. Hence, this suggests that individuals may exploit the affordances of any kind of technology to take charge and control regardless of whether it is new or low tech.

The extent to which low or new tech tools promote collaboration in group settings, therefore, would appear to be a function of the perceived or implied ownership and whether gatekeeping can be prevented. While previous research has shown how shared surfaces, namely tabletops can increase group awareness and encourage more equitable participation in design tasks and collaborative games [e.g., 14], it is less clear whether such benefits can accrue in more open-ended meetings, such as breakout sessions, where the participants are not necessarily familiar with the technology, the outputs of the task or each other.

Another factor that can affect participation levels is the size of the group. In our study, the workshop organizers had decided to split the workshop into 4 breakout groups, comprising 9-10 people. With the exception of Group A, the large size made it difficult for all group members to use the surface they had been allocated. Smaller groups at the tabletop or the writeable wall might have enabled them to remain as one unit and maintain awareness of each other rather than having to further divide into sub-groups. While keeping the size of the groups small in breakout sessions is considered optimal [21], the workshop organizers chose not to do so here, partly because of the constraints and the availability of the technology in the QSpace. It would be interesting to see whether more equitable participation and less gatekeeping would happen with smaller sized breakout groups.

An important consideration that needs further research is the permanent and public nature of the interactions that result when using different technologies. Whereas a post-it note written on can be easily discarded, a wall cannot. It needs a special kind of cloth and liquid solution to erase it, which often is not ready to hand. This can affect how easily or comfortable someone is when writing on a wall. Likewise, a node filled in on a software tool is perceived as being permanent, especially if the participation does not know how to delete it. The act of writing is also private on a post-it note and the person can decide whether to make it public or not; whereas at the tabletop it is public from the beginning.

In sum, while the writing is not on the walls for tabletops (or writeable walls for that matter) it is important to consider what their added value is in more open-ended meetings where there may be considerable social awkwardness and uncertainty as to what a group member can and cannot do. Having a facilitator in a group may help group member's overcome these barriers although he/she may, too, find it difficult to resist turning into a gatekeeper.

Acknowledgments. We thank all the participants, the facilitators of the space and the organizers of the workshop for making that study possible. 


\section{References}

1. Agamanolis, S.: Designing displays for Human Connectedness. In: O’Hara, K., Perry, M., Churchill, E., Russell, D. (eds.) Public and situated displays: Social and interactional aspects of shared display technologies, pp. 309-335. Kluwer Academic, Dordrecht (2003)

2. Bailey, B.P., Konstan, J.A., Carlis, J.V.: Demais: Designing multimedia applications with interactive storyboards. In: Proceedings of ACM Multimedia, pp. 241-250. ACM Press, New York (2001a)

3. Barthelmess, P., Kaiser, E., Lunsford, R., McGee, D., Cohen, P., Oviatt, S.: Humancentered collaborative interaction. In: Proceedings of the 1st ACM international Workshop on Human-Centered Multimedia, HCM 2006, Santa Barbara, California, USA, October 27, 2006, pp. 1-8. ACM, New York (2006)

4. Brignull, H., Izadi, S., Fitzpatrick, G., Rogers, Y., Rodden, T.: The introduction of a shared interactive surface into a communal space. In: Proceedings of CSCW 2004: Conference on Computer Supported Cooperative Work, ACM, New York (2004)

5. Brignull, H., Rogers, Y.: Enticing people to interact with large public displays in public spaces. In: Proceedings of INTERACT 2003: Ninth IFIP TC13 International Conference on Human-Computer, IOS Press, Zurich (2003)

6. Churchill, E.F., Nelson, L., Denoue, L., Girgensohn, A.: The Plasma Poster Network: Posting multimedia content in public places. In: Proceedings of INTERACT 2003: Ninth IFIP TC13 International Conference on Human-Computer. IOS Press, Zurich (2003)

7. Debategraph, http: / / debategraph.org

8. Dietz, P., Leigh, D.: DiamondTouch: A multi-user touch technology. In: Proceedings of the 14th Annual ACM Symposium on User interface Software and Technology. UIST 2001, pp. 219-226. ACM, New York (2001)

9. Eden, H., Hornecker, E., Scharff, E.: Multilevel design and role play: experiences in assessing support for neighbourhood participation in design. In: Proceedings of DIS 2002, pp. 387-392. ACM, London (2002)

10. Gorman, P., Ash, J., Lavelle, M., Lyman, J., Delcambre, L., Maier, D.: Bundles in the Wild: Managing Information to Solve Problems and Maintain Situation Awareness. Library Trends 49(2), 266-289 (2000)

11. Grasso, A., Muehlenbrock, M., Roulland, F., Snowdon, D.: Supporting communities of practice with large screen displays. In: O'Hara, K., Perry, M., Churchill, E., Russell, D. (eds.) Public and situated displays: Social and interactional aspects of shared display technologies, pp. 261-283. Kluwer Academic, Dordrecht (2003)

12. Hammersley, M., Atkinson, P.: Ethnography: Principles in practice, 3rd edn. Routledge, London (2007)

13. Heath, C., Luff, P.: Technology in Action. In: Pea, R., Brown, J.S., Heath, C. (eds.) Learning in Doing: Social, Cognitive and Computational Perspectives, Cambridge University Press, Cambridge (2000)

14. Hornecker, E., Marshall, P., Dalton, N., Rogers, Y.: Collaboration and Interference: Awareness with Mice or Touch Input. In: Proceedings of ACM CSCW Conference 2008, pp. 167-176. ACM Press, New York (2008)

15. Huang, E.M., Mynatt, E.D., Trimble, J.P.: When design just isn't enough: The unanticipated challenges of the real world for large collaborative displays. Personal and Ubiquitous Computing, Special Issue on Ubiquitous Computing in the Real World 11(7), 537-547 (2007)

16. Mackay, W.E.: Is paper safer? The role of flight strips in air traffic control. ACM Transactions on Computer- Human Interaction 6(4), 311-340 (1999) 
17. Marshall, P., Hornecker, E., Morris, R., Dalton, N., Rogers, Y.: When the fingers do the talking: A Study of Group Participation With Varying Constraints to a Tabletop Interface. In: Proceedings of IEEE Tabletops and Interactive Surfaces, pp. 37-44. IEEE, Los Alamitos (2008)

18. McDonald, D.W., McCarthy, J.F., Soroczak, S., Nguyen, D.H., Rashid, A.M.: Proactive displays: Supporting awareness in fluid social environments. ACM Transactions on Computer- Human Interaction, 14(4), Article 16 (2008)

19. McGee, D.R., Cohen, P.R., Wu, L.: Something from nothing: Augmenting a paper-based work practice with multimodal interaction. In: Proceedings of the Conference on Designing Augmented Reality Environments, Helsingor, Denmark, April 12-14, 2000, pp. 71-80. ACM Press, New York (2000)

20. Microsoft Surface, http: / / www. microsoft. com/SURFACE/index.html

21. Muller, G.: Workshop How to To (2008), http: //www.gaudisite.nl/workshophowtopaper.pdf

22. Mynatt, E.D., Igarashi, T., Edwards, W.K., LaMarca, A.: Flatland: New dimensions in office whiteboards. In: Proceedings of the SIGCHI Conference on Human Factors in Computing Systems: the CHI Is the Limit, Pittsburgh, Pennsylvania, United States, May 15-20, 1999, pp. 346-353. ACM, New York (1999)

23. O'Hara, K., Glancy, M., Robertshaw, S.: Understanding collaborative play in an urban screen game. In: Proceedings of CSCW 2008: The 2008 ACM Conference on Computer Supported Cooperative Work. ACM, San Diego (2008)

24. Pantidi, N., Robinson, H.M., Rogers, Y.: Can technology-rich spaces support multiple uses? In: Proceedings of HCI2008: The 22nd annual conference of Interaction, a specialist group of the British Computer Society. BCS, Liverpool (2008)

25. Pedersen, E.R., McCall, K., Moran, T.P., Halasz, F.G.: Tivoli: An electronic whiteboard for informal workgroup meetings. In: Proceedings of the INTERACT 1993 and CHI 1993 Conference on Human Factors in Computing Systems, Amsterdam, The Netherlands, April 24 - 29, 1993, pp. 391-398. ACM, New York (1993)

26. Peltonen, P., Kurvinen, E., Salovaara, A., Jacucci, G., Ilmonen, T., Evans, J., Oulasvirta, A., Saarikko, P.: It's mine, don't touch!: Interactions at a large multi-touch display in a city centre. In: Proceedings of CHI 2008: Conference on Human Factors in Computing Systems. ACM, Florence (2008)

27. Preece, J., Rogers, Y., Sharp, H.: Interaction Design: Beyond Human-Computer Interaction, 2nd edn. John Wiley \& Sons, New York (2007)

28. Rogers, Y., Lim, Y., Hazlewood, W.R., Marshall, P.: Equal Opportunities: Do Shareable Interfaces Promote More Group Participation than Single User Displays? To Appear in Human Computer Interaction (2008)

29. Rogers, Y., Lindley, S.: Collaborating around vertical and horizontal displays: which way is best? Interacting With Computers 16, 1133-1152 (2004)

30. Russell, D.M., Sue, A.: Large interactive public displays: Use patterns, support patterns, community patterns. In: O’Hara, K., Perry, M., Churchill, E., Russell, D. (eds.) Public and situated displays: Social and interactional aspects of shared display technologies, pp. 3-18. Kluwer Academic, Dordrecht (2003)

31. Sacks, H., Schegloff, E.A., Jefferson, G.: A simplest systematics for the organization of turn taking in conversation. Language 50(4), 696-735 (1974)

32. Sellen, A.J., Harper, R.H.R.: The Myth of the Paperless Office. MIT Press, Cambridge (2002)

33. Sierhuis, M.: Collaboratively Modeling Mission Control at NASA (2006), http: / / news . kmi . openac.uk/rostra/news.php? $r=55 \& t=2 \& i d=20 \#$

34. Whittaker, S., Schwarz, H.: Meetings of the Board: The Impact of Scheduling Medium on Long Term Group Coordination in Software Development. Computer Supported Cooperative Work 8(3), 175-205 (1999) 